\title{
FGF21 mediates the anti-depressant effects of exercise by coordinating the crosstalk between central and peripheral organs
}

\author{
Yan Liu ${ }^{1,2}$, Suk Yu Yau ${ }^{3}$, Qingning Liang ${ }^{4}$, Yao Wang ${ }^{1,2}$, Aimin $\mathrm{Xu}^{1,2,5 *}$
}

Exercise is an effective anti-depressant treatment; however, its underlying mechanism remains largely unknown. Fibroblast growth factor 21 (FGF21) is a metabolic hormone critically involved in energy metabolism. Here, we showed that FGF21 knockout significantly diminished exercise-elicited anti-depressant effects, while replenishment with recombinant FGF21 effectively restored the effects of exercise on alleviation of depression by suppressing neuroinflammation, enhancing adult neurogenesis and synaptic plasticity in the hippocampus. However, the FGF21 co-receptor $\beta$-klotho was not expressed in hippocampal neurons. The anti-depressant property of FGF21 was attributed to its ability to stimulate adipocyte secretion of adiponectin, which functioned as a downstream effector of FGF21 to confer the anti-depressant effects. Collectively, these data identify FGF21 as an important player in mediating the anti-depressant effects of exercise, possibly by coordinating multi-organ crosstalk among liver, adipose tissue and brain, and also raise the possibility that FGF21 and its agonists may represent a promising therapeutic approach for depression.

Keywords: depression, exercise, FGF21, obesity, metabolic disorders

\section{INTRODUCTION}

Depression is one of the leading causes of disability and mortality worldwide. The global prevalence of depression is consistently increasing in recent decades (1). Lifetime prevalence estimates of depression range from $20-25 \%$ in women and $7-12 \%$ in men $(1)$. Unfortunately, there is currently no effective therapy available for depression, partly due to a considerable clinical heterogeneity and a lack of defined etiology. Several mechanisms have been proposed as important contributors to the pathogenesis of depression, such as impairment in hippocampal neurogenesis and plasticity, activation of neuroinflammation and astrocyte, and imbalance in neurotransmitters $(2,3)$. Notably, the prevalence of depression is higher in individuals with obesity compared to those with normal body mass index (4). Epidemiological research from both cross-sectional and longitudinal studies has confirmed a positive association and the existence of a bidirectional relationship between obesity and depression (5). Mechanistic studies have also established several shared and interconnected pathophysiological mechanisms between these two common conditions, including increased cortisol, oxidative and nitrate stress and chronic low grade inflammation (6). Therefore, targeting metabolic disorders might be an alternative approach for the prevention and treatment of depression.

Exercise is known as the cornerstone for the management of several chronic diseases, including obesity, diabetes and depression. A large number of previous studies have documented the therapeutic effects of exercise in treating depressive disorders through multiple effects on promoting neurogenesis and neuroplasticity, reversing altered neurotransmitters, stimulating the production of neurotropic factors and suppressing neuroinflammation (2, 7). More recently, adiponectin, an adipocyte-derived adipokine with insulinsensitising and anti-diabetic effects (8), has been found to play a key role in mediating the anti-depressant effects of exercise in mice through the activation of AMP-activated protein kinase (AMPK) signaling pathway $(2,9)$. However, the underlying mechanisms involved in the functional benefits of exercise on depression still

\footnotetext{
${ }^{1}$ State Key Laboratory of Pharmaceutical Biotechnology, The University of Hong Kong Hong Kong SAR, China. ${ }^{2}$ Department of Medicine, The University of Hong Kong, Hong Kong SAR, China. ${ }^{3}$ Department of Rehabilitation Science, The Hong Kong Polytechnic University, HKSAR, China. ${ }^{4}$ Ma Man Kei and Lo Pak Sam College, University of Macau Macau SAR, China. ${ }^{5}$ Department of Pharmacology and Pharmacy, The University of Hong Kong, Hong Kong SAR, China.

*Corresponding author. Email: amxu@hku.hk (A.X.)
}

remain largely unknown.

Fibroblast growth factor 21 (FGF21), a hormone secreted predominately from the liver, plays an important role in regulating energy homeostasis and possesses multiple therapeutic benefits against obesity-related diseases (10). Administration of recombinant FGF21 to diabetic rodents and primates reduces body weight, increases insulin sensitivity, improves glucose and lipid profiles (10), and inhibits pancreatic inflammation and islet hyperplasia (11). Due to the lack of heparin-binding activity, FGF21 exerts its biological effects by binding to a receptor complex between the FGF receptor-1 (FGFR1) and its co-receptor $\beta$-Klotho, which is a single transmembrane glycoprotein that interacts with the carboxyl terminus of FGF21 and determines the tissue specificity of FGF21 actions (12). In particular, both FGFR1 and $\beta$-Klotho are highly expressed in adipose tissue, where FGF21 enhances glucose uptake, inhibits lipolysis and promotes both expression and secretion of adiponectin (8). FGF21 induces adiponectin secretion in a peroxisome proliferator-activated receptor $\gamma$-dependent manner, while adiponectin functions as an indispensable downstream effector of FGF21 in mediating the systemic effects of FGF21 on insulin sensitivity and glucose homeostasis (8). Furthermore, the anti-atherosclerotic activity of FGF21 was partly dependent on its ability to stimulate adiponectin secretion in adipocytes (13). Notably, both FGFRs and $\beta$-Klotho have been found to be abundantly expressed in several brain regions, such as the hypothalamus and the cortex (14), suggesting that in addition to the peripheral actions, FGF21 may have some central effects.

Recently, FGF21 is found to be present in the cerebrospinal fluid of both humans (15) and fasted mice (14), suggesting that FGF21 could pass through the blood-brain barrier (BBB) and plays a role in the brain. Moreover, clinical studies have reported a lower level of plasma FGF21 in patients with depression, which can be increased by treatment with anti-depressant drugs (16). A recent study has also shown that FGF21 treatment alleviates anxiety in obese mice mainly through improving its peripheral metabolism (17). However, the specific organs responsible for the FGF21 actions and the molecular mechanisms by which FGF21 mediates the anti-depressant effects of exercise remain poorly understood at this stage.

In the present study, we investigated the potential role of FGF21 in mediating the anti-depressant effects of physical exer- 
cise in rodent models using both gain- and loss-of functional approaches, and examined the mechanisms underlying the antidepressant effects of FGF21.

\section{RESULTS}

\section{FGF21 increases in response to acute treadmill training}

To evaluate the relevance of FGF21 in exercise-induced antidepressant effects, adult mice were subjected to treadmill training to see whether circulating FGF21 would increase in response to exercise training. Serum FGF21 was found to increase along with exercise training and peaked 3 hours after the start of training (Fig. 1B), which was approximately 1-2 hours later than the peak of free fatty acids (FFA) (Fig. 1A), suggesting that similar to fasting, increased FFA might be the natural agonist of peroxisome proliferator-activated receptor alpha that induces FGF21 production during exercise. Furthermore, exercise-induced elevation of circulating FGF21 was produced predominately from the liver but not the soleus muscle (Fig. 1C-E).
A

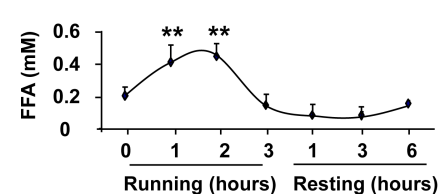

C

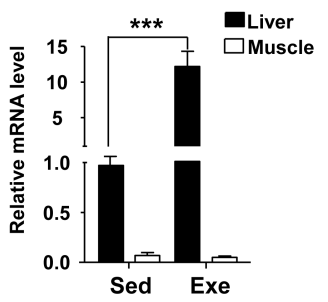

E

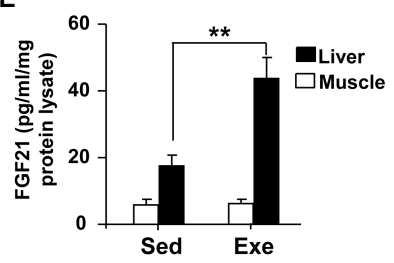

B

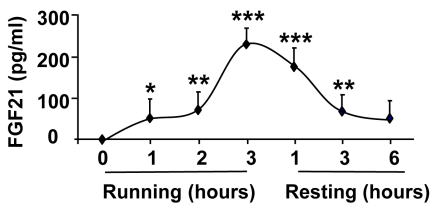

D

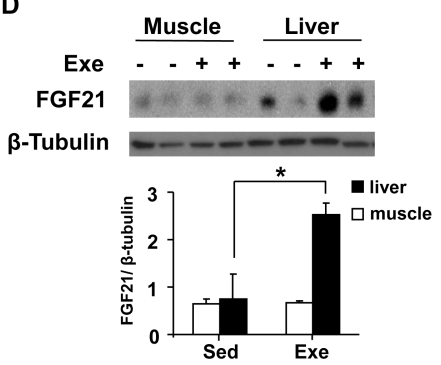

Fig. 1. Circulating FGF21 increases in response to exercise training. Ten-weekold $\mathrm{C} 57 \mathrm{BL} / 6 \mathrm{~J}$ mice were subjected to acute exercise with treadmill (Exe) or nonrunning treatment (Sed). (A) Serum levels of free fatty acids (FFA) and (B) FGF21 were determined at different time points during running and resting. (C) The mRNA expression of FGF21 in soleus muscle and liver under sedentary state or after exercise was determined by real-time PCR. The protein abundance of FGF21 in muscle and liver under sedentary state or after exercise was determined by (D) western blotting analysis and (E) ELISA. Exercise (Exe) vs sedentary control (Sed); $n=6$ mice per group. Data are presented as mean $\pm \mathrm{SEM}^{*} p<0.05,{ }^{* *} p<0.01,{ }^{* * *} p<0.001$.

\section{FGF21 is an obligatory mediator for the anti-depressant effects of exercise}

Next, we investigated whether FGF21 would be required for the anti-depressant effects of exercise in an obese mouse model showing depressive phenotype induced by high-fat diet (HFD). Both FGF21 knocked-out (KO) mice and wild-type (WT) littermates were fed HFD for eight weeks to induce obesity before subjected to treadmill training for another four weeks, while sedentary mice (non-runners) were used as controls. After the treadmill training, a multitude of behavioural tests was performed to assess the effects of exercise on the alleviation of depression-like behaviours (Fig. 2A). FGF21 knockout in non-runners did not affect immobility time in forced swimming test (FST) when compared to the WT non-runners. In contrast, treadmill training significantly decreased immobility time in WT but not in FGF21 KO mice, suggesting that FGF21 knockout diminished the anti-depressant effects of treadmill running (Fig. 2B). Similar results were also observed in the tail suspension test (TST), treadmill running significantly reduced the immobility time in WT runners when compared to WT non-runners, whereas FGF21 KO mice showed no improvement in immobility after treadmill training (Fig. 2C). Treadmill training also significantly increased sucrose consumption in the sucrose preference test (SPT) in WT but not FGF21 KO mice (Fig. 2D), indicating that FGF21 knockout abolished running-induced mitigation of anhedonia. Moreover, treadmill training significantly increased the time spent in the central area (Fig. 2E) and the number of social interactions (Fig. 2F) in WT but not FGF21 KO mice. Collectively, these results suggest that FGF21 deficiency mitigates the beneficial effects of physical exercise on depressionlike behaviours.

\section{A}

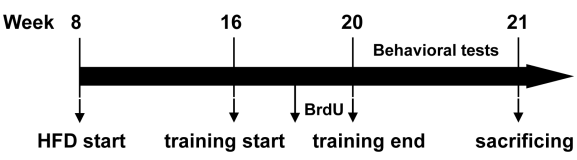

B

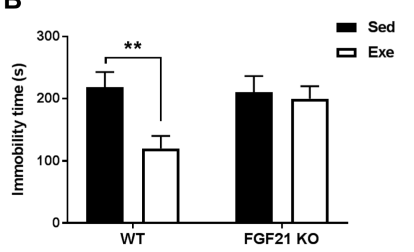

C

D
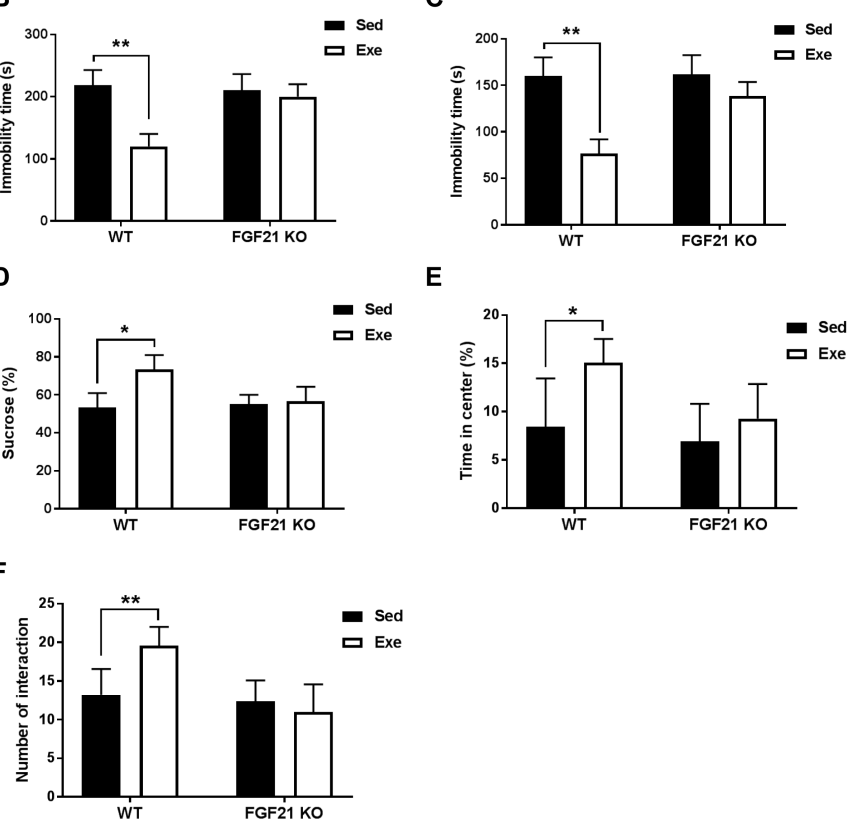

Fig. 2. FGF21 is obligatory for exercise-induced alleviation of depression-like behaviours. (A) Experimental timeline for exercise training and behavioural tests. FGF21 KO mice and WT littermates received exercise training (Exe) or sedentary lifestyle (Sed) for four weeks, followed by behavioural tests. (B) TST and (C) FST assessed the duration of immobility that parallels depressive severity. (D) SPT examined the loss of preference to sucrose (anhedonia), a core symptom in depression. (E) Time spent in the central area and (F) number of social interactions; low values correspond to core features of anxiety. Data are presented as mean \pm SEM; ${ }^{*} p<0.05,{ }^{* *} p<0.01 ; n=8$ mice per group.

To further confirm the indispensable role of FGF21 in exerciseinduced anti-depressant effects, we examined whether subcutaneous replenishment with recombinant mouse FGF21 (rmFGF21) could restore the anti-depressant effects of exercise in FGF21 KO mice (Fig. 3A). Compared to saline control, rmFGF21 sup- 
plementation significantly alleviated depression-like behaviours in FGF21 KO mice as evidenced by decreased immobility time in TST (Fig. 3B) and FST (Fig. 3C), increased consumption of sucrose water (Fig. 3D), prolonged time spent in the central area (Fig. 3E) and increased number of social interactions (Fig. 3F). Taken together, these data demonstrate that rmFGF21 supplementation alone could mimic the anti-depressant effects of exercise and reverse depression-like behaviours.

\section{A}

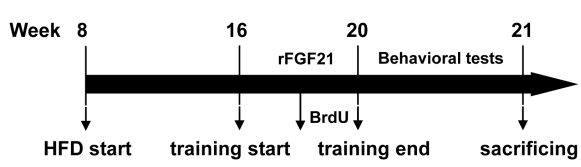

B

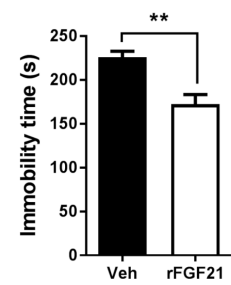

E

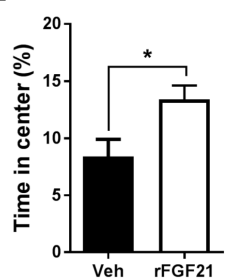

C

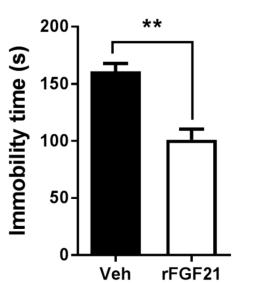

$\mathbf{F}$

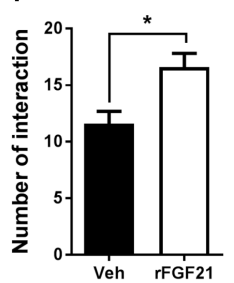

D

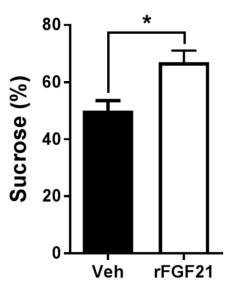

A

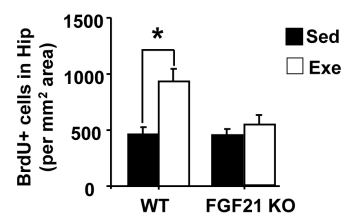

C

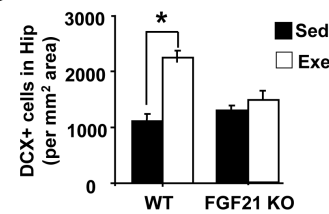

B

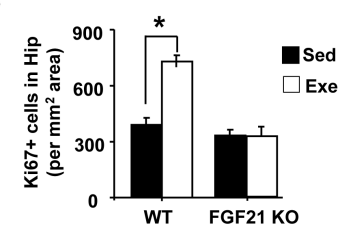

Fig. 4. FGF21 enhances neurogenesis in the hippocampus in response to exercise training. WT and FGF21 KO mice were injected with BrdU to label newborn cells during the last five consecutive days of the four-week training period. Quantification of (A) $\mathrm{BrdU}^{+}$cells (B) $\mathrm{Ki}^{+}$cells and (C) $\mathrm{DCX}^{+}$cells in the hippocampus. Data are presented as mean $\pm \mathrm{SEM} ; *<0.05 ; n=8$ mice per group.

\section{FGF21 suppresses neuroinflammation and promotes synaptic} plasticity in the hippocampus

We investigated whether the behavioural alterations in exercised FGF21 KO mice were linked to corresponding changes in neuroinflammation and synaptic plasticity in the hippocampus. While running significantly reduced pro-inflammatory markers, including monocyte chemoattractant protein-1 (MCP-1), tumour necrosis factor- $\alpha$ (TNF- $\alpha$ ), interleukin- $\beta$ (IL- $1 \beta$ ) and interleukin-6 (IL6 ) in the hippocampus of WT mice, such reduction was largely compromised in FGF21 KO mice (Fig. 5A). In contrast, running did not significantly affect the levels of anti-inflammatory markers (IL4 and IL10) in either WT or FGF21 KO mice (Fig. 5B). Moreover, expressions of the macrophage/microglia activity markers, including ionised calcium-binding adaptor molecule (IBA1), cyclindependent kinase $11 \mathrm{~b}(\mathrm{CD} 11 \mathrm{~b})$ and macrophage inflammatory protein $1 \alpha(\mathrm{MIP} 1 \alpha)$ were not significantly altered after exercise training in both WT and FGF21 KO mice (Fig. 5C), suggesting that FGF21 exerts its anti-depressant effects mainly through the inhibition of neuroinflammation. In addition, expression levels of proteins mediating synaptic plasticity calcium/calmodulindependent protein kinase type II subunits alpha and beta (CamKII $\alpha$ and $\operatorname{CamKII} \beta$ ) and $\mathrm{ARC}$, as well as the AMPA receptor subunit glutamate A1 (GluA1), were significantly increased after exercise training in WT but not FGF21 KO mice (Fig. 5D). Taken together, these results suggest that FGF21 mediates the anti-depressant effects of exercise through enhancing adult neurogenesis and synaptic plasticity, whilst suppressing neuroinflammation.

\section{The hippocampus is unlikely to be the direct target of FGF21}

We $(14)$ and others $(18,19)$ have previously demonstrated that FGF21 could cross the BBB and is present in cerebrospinal fluid in both humans and rodents. Animal-based studies have also suggested that FGF21 could exert direct effects in the brain, especially in the hypothalamic neurons to control systemic metabolism and reproduction activities $(14,18)$. However, whether it could pass the BBB and act directly in the hippocampus to mediate the antidepressant effects of exercise remain obscure. We first investigated whether FGF21 receptor complex could be detected in the hippocampus. Expression levels of FGFR1 in the hippocampus was comparable to those in hypothalamus at both mRNA and protein levels (Fig. 6A and 6B respectively). However, neither mRNA 

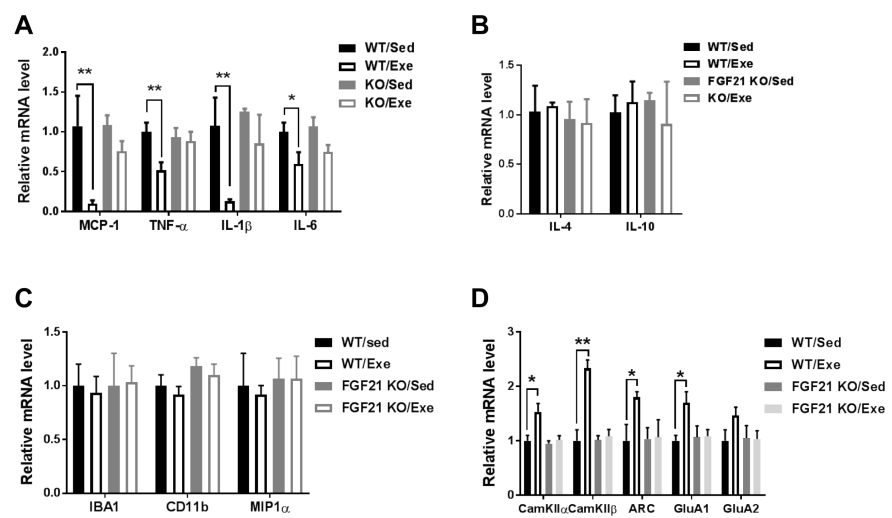

Fig. 5. FGF21 suppresses neuroinflammation and promotes synaptic plasticity in hippocampus. WT and FGF21 KO mice received exercise training (Exe) or sedentary lifestyle (Sed) for four weeks. Hippocampus was subjected to real-time PCR analysis for determining the expression levels of markers for (A) inflammation, (B) antiinflammation, (C) microglia activity and (D) synaptic plasticity. Data are presented as mean $\pm \mathrm{SEM} ;{ }^{*} p<0.05,{ }^{*} p<0.01 ; n=8$ mice per group. MCP-1: monocyte chemoattractant protein-1; TNF- $\alpha$ : tumour necrosis factor $\alpha$; IL- $1 \beta$ : interleukin- $1 \beta$; IL-6: interleukin-6; IBA1: ionised calcium-binding adaptor molecule; CD11b: cyclindependent kinase $11 \mathrm{~b}$; MIP1 $\alpha$ : macrophage inflammatory protein $1 \alpha$; CamKII $\alpha$ and CamKII $\beta$ : calcium/calmodulin-dependent protein kinase type II subunits alpha and beta; GluA1 and GluA2: AMPA receptor subunits glutamate $A 1$ and $A 2$.

nor protein expression of $\beta$-Klotho, the critical co-receptor for FGF21 actions, could be detected in the hippocampus (Fig. 6C and 6B respectively), suggesting that the FGFR1- $\beta$-Klotho complex was undetectable in the hippocampus. Collectively, considering the dependence of FGF21 on its receptor complex to exert biological effects, our results suggest that FGF21 may act indirectly on the hippocampus to mediate its anti-depressant effects of physical exercise.

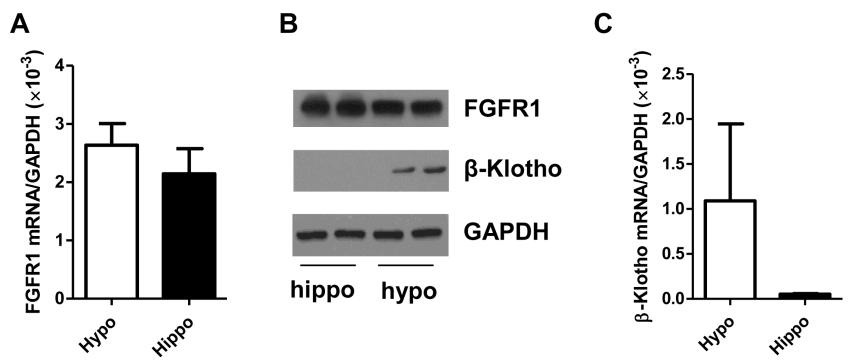

Fig. 6. The FGF21 co-receptor $\beta$-klotho is not expressed in the hippocampus Hippocampus (Hippo) and hypothalamus (Hypo) from ten-week-old male C57BL/6 mice $(n=5)$ were isolated and subjected to quantification of mRNA expression levels of (A) FGFR1 and (C) $\beta$-Klotho by real-time PCR analysis. (B) Protein levels of FGFR1 and $\beta$-Klotho were determined by western blot analysis. Data are expressed as mean \pm SEM.

\section{Adiponectin serves as a downstream effector for the anti-depressant effects of FGF21}

Considering that adiponectin serves as an obligatory downstream target of FGF21 to control metabolic homeostasis (8) and that it has previously been shown to elicit neurogenesis through the activation of AMPK in the hippocampus (2), we continue to explore whether adiponectin also serves as a downstream mediator of FGF21 to confer the anti-depressant effects of exercise. FGF21 KO mice were fed with HFD for eight weeks to induce obesity and then had recombinant mouse adiponectin or saline (as vehicle control) chronically delivered by osmotic pumps as previously described (8). A series of behavioural tests were carried out to evaluate the role of adiponectin in mediating FGF21's central action (Fig. 7A). Increasing adiponectin levels in FGF21 KO mice by osmotic pump infusion significantly reduced immobility time in FST (Fig. 7B) and TST (Fig. 7C) compared to mice infused with saline. Additionally, adiponectin infusion also significantly increased the consumption of sucrose in FGF21 KO mice compared to those infused with vehicle (Fig. 7D), suggesting that increasing adiponectin levels is sufficient in inducing the anti-depressant effects of exercise even in the absence of FGF21. Moreover, infusion with adiponectin also significantly increased the time spent in the central area (Fig. 7E) and in the number of social interactions (Fig. 7F) in FGF21 KO mice. Taken together, these results suggest that adiponectin could reverse the depression-like behaviours caused by FGF21 deficiency.

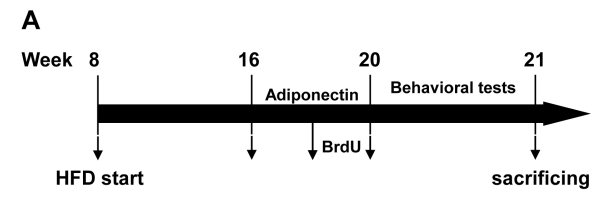

B

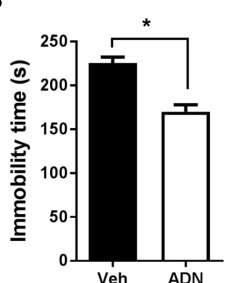

C
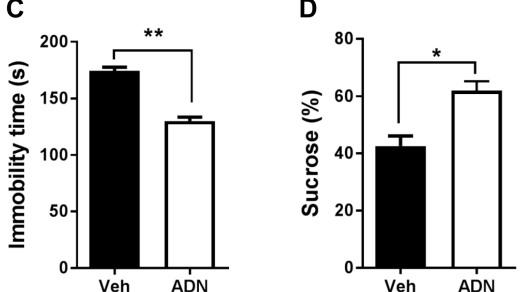

E

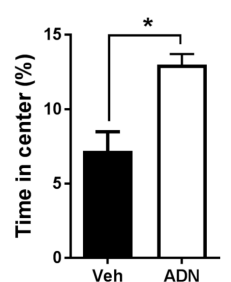

$\mathbf{F}$

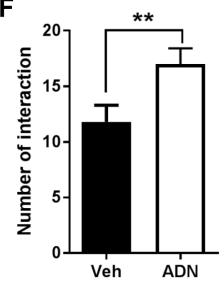

Fig. 7. Replenishment with recombinant adiponectin reverses the depression-like behaviours in FGF21 knocked-out mice. (A) Experimental timeline for adiponectin infusion and behavioural tests. FGF21 KO mice received recombinant adiponectin (ADN) or saline as vehicle (Veh) for four weeks, followed by behavioural tests. (B) TST and (C) FST assessed the duration of immobility that parallels depressive severity. (D) SPT examined the loss of preference to sucrose (anhedonia), a core symptom in depression. (E) Time spent in the central area and (F) number of social interactions; low values correspond to core features of anxiety. Data are presented as mean \pm SEM; ${ }^{*} p<0.05,{ }^{* *} p<0.01 ; n=8$ mice per group.

We subsequently investigated whether increasing adiponectin levels in FGF21 KO mice was correlated to its capacity to mitigate neuroinflammation and promote neurogenesis. FGF21 KO mice infused with recombinant adiponectin showed a significant reduction of inflammatory markers in hippocampus compared to those infused with vehicle (Fig. 8A). However, increasing adiponectin levels by osmotic pump infusion did not significantly affect the levels of anti-inflammatory markers (Fig. 8B) and microglial activation markers (Fig. 8C) in mice. Consistent with the improvement in depression-like behaviours, infusing adiponectin significantly increased the transcription of proteins involved in synaptic plasticity, except for GluA2 (Fig. 8D). Furthermore, infusing adiponectin in FGF21 KO mice significantly increased the number of $\mathrm{BrdU}^{+}$cells (Fig. 8E). Collectively, these data suggest that adiponectin is an obligatory downstream effector for FGF21 in mediating the anti-depressant effects of exercise. 


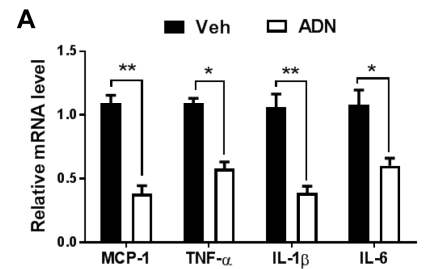

C
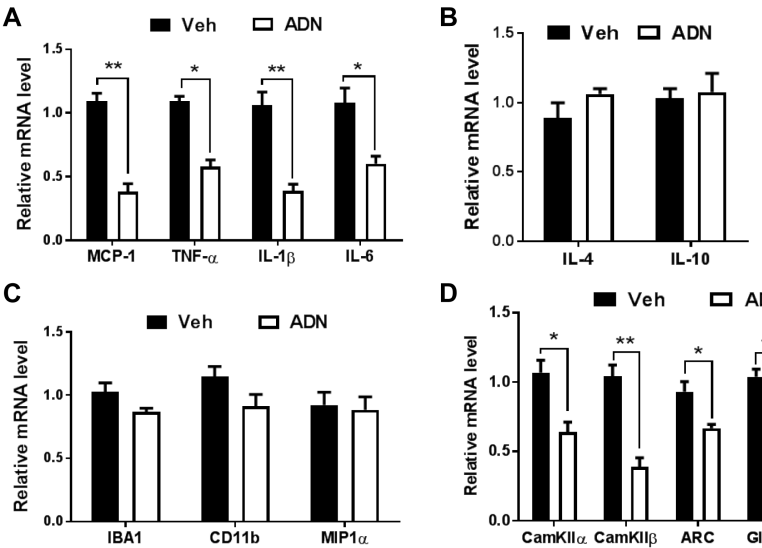

D

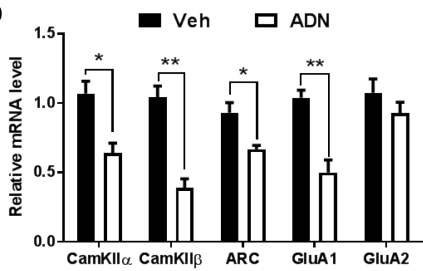

E

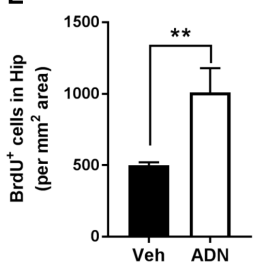

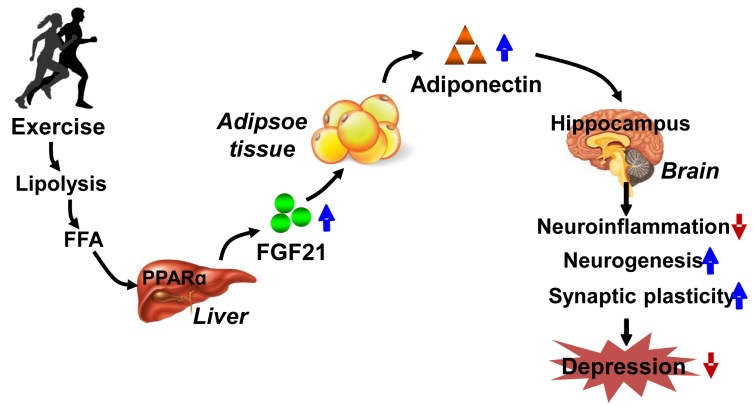

Fig. 9. Proposed mechanism whereby the FGF21-adiponectin axis confers the antidepressant effects of exercise by mediating inter-organ crosstalk. In response to exercise, FGF21 produced from the liver travels to adipose tissue to induce the secretion of adiponectin, which in turn passes through BBB to directly act on the hippocampus to suppress neuroinflammation and promote neurogenesis and synaptic plasticity, thus reducing depression-like symptoms. FFA: free fatty acids; $\operatorname{PPAR} \alpha$ : peroxisome proliferator-activated receptor $\alpha$.

as an Akt-regulated myokine, the source of exercise-induced FGF21 has long been controversial. Contrary to the observation that expression and secretion of FGF21 were upregulated in $\mathrm{C} 2 \mathrm{C} 12$ myocytes (22), we observed that exercise significantly augmented the expression and secretion of FGF21 in the liver, whereas it remained unchanged in the skeletal muscles. Our results further reinforce the notion that hepatic FGF21 is a major source of this molecule contributing to increased serum levels after exercise, suggesting a critical role of hepatokines in the mediation of multiple benefits of physical exercise.

In addition to the well-established role of FGF21 in peripheral metabolism, neuroprotective effects of FGF21 have also been reported in several clinical studies. FGF21 levels in cerebrospinal fluid were found to be negatively related to the scores of Beck Depression Inventory in male participants (23). Additionally, circulating levels of FGF21 were found to be positively correlated with the abilities to cope with stress after chronic exposure (24). Moreover, the magnitude of increase in serum FGF21 levels was found to be associated with the treatment effects of antidepressant drugs (16), though no significant correlation had been observed in female participants (23). Despite the inconsistencies in clinical findings possibly due to the heterogeneity of participants and experimental designs across different studies, our animal research showed that FGF21 knockout diminished improvement in depression-like behaviours induced by exercise, as evidenced by sustained activation of neuroinflammation, deficient neurogenesis and impaired synaptic plasticity in the hippocampus. Replenishment with recombinant FGF21 into FGF21 KO mice without exercise further showed that increasing FGF21 alone mimicked the beneficial effects of exercise in obese mice. Taken together, our results demonstrated that FGF21 is indispensible for exercise to ameliorate depression, suggesting that both FGF21 and its agonists may mimic the effects of physical exercise for treating depressive disorders.

The crucial role of hippocampus in the regulation of mood has been well established. Adult hippocampal neurogenesis has been reported to be negatively affected by aging and chronic stresses such as oxidative damage, neuroinflammation and cerebral metabolic dysfunction (25). In line with previous reports that hippocampal neurogenesis served as a therapeutic target of several anti-depressant drugs, we also observed a significant increase in the number of newborn cells and ameliorated depression severity in mice after four weeks of treadmill training. However, 
such alleviation in depressive symptoms was largely abolished in FGF21 KO mice, which could be reverted by recombinant FGF21 treatment, suggesting that anti-depressant effects of FGF21 involve amelioration of hippocampal dysfunction. Due to the lack of heparin-binding activity, the multipleiotropic metabolic effects of FGF21 are dependent on the tissue-specific expression of FGFR1 and $\beta$-Klotho, the obligatory receptor complex for FGF21 (12). Therefore, we first examined the expression of this receptor complex in hippocampus to determine whether FGF21 mediates the anti-depressant effects of exercise through acting directly on hippocampus. To our surprise, though abundantly expressed in hypothalamus, $\beta$-Klotho could hardly be detected in hippocampus, suggesting that FGF21 cannot act directly on hippocampus to exert its anti-depressant effects. Both we (8) and others (26) have previously demonstrated that the metabolic benefits of FGF21 on glucose homeostasis and insulin sensitivity are partly dependent on adiponectin, suggesting that adiponectin functions as a key downstream effector for FGF21. Therefore, we speculate that adiponectin mediates the indirect effects of FGF21 on hippocampus. Studies from both humans and animal models have shown that both acute and chronic exercise increased circulating adiponectin levels $(27,28)$ and that the magnitude of its increase was positively correlated with its improvement in metabolic homeostasis (28). A recent finding from our group also indicated an indispensable role of adiponectin in mediating the anti-depressant effects of exercise through the activation of adiponectin receptor 1/AMPK signalling pathways in the hippocampus (2). Consistently, we found that chronic treatment with recombinant adiponectin without exercise training alleviated depression in obese mice in the absence of FGF21. This ameliorating effect was accompanied by reduction in neuroinflammation markers and enhancement in neurogenesis and synaptic plasticity.

Obesity, which has reached epidemic proportions globally, is often comorbid with depression. The latter is projected to be the second leading cause of disability (29). Together, they pose a heavy burden to public health. Considering the close correlation between obesity and depression, and the potent effect of FGF21 on metabolic homeostasis, our findings suggest that targeting peripheral metabolism might provide an effective alternative approach for treating depressive symptoms. With a rapid response to exercise challenge, our results also provide the possibility that FGF21 could be used as a biomarker for quantitative assessment of the anti-depressant effects of exercise or even in the synthesis of new anti-depressant drugs. Medical practitioners may also use FGF21 as an indicator to monitor appropriate intensity and duration of exercise for each patient with diverse severity of depression. Furthermore, since many pharmaceutical companies are developing FGF21 agonists for treating metabolic diseases, the demonstration of its anti-depressant effects of FGF21 by our study will also expand its therapeutic applications for mental diseases and provide alternative therapeutic approach for patients with depression, especially those with relapsing depressive disorders.

In summary, though our study was mainly based on murine models and its translation into humans warrants further investigation through randomised controlled clinical trials, our results identify FGF21 as an obligatory mediator for the anti-depressant effects of physical exercise through the crosstalk among liver, adipose tissue and brain. FGF21 suppresses neuroinflammation and promotes adult neurogenesis and synaptic plasticity in the hippocampus in an adiponectin-dependent manner. These findings delineate a novel role of FGF21 in anti-depressant effects of physical exercise and suggest that targeting FGF21 could be a potential therapeutic approach for obesity-related depression.

\section{MATERIALS AND METHODS}

\section{Animal preparation}

All experimental procedures were approved by the committee on the use of live animals for teaching and research of the University of Hong Kong and were carried out in accordance with the guide for the care and use of laboratory animals. All mice (FGF21 KO and wild-type littermates, and C56BL/6J mice) were maintained on 12-hour light and dark cycles under controlled environment settings (temperature: $23 \pm 1^{\circ} \mathrm{C}$ ), with free access to food and water. Eightweek-old male mice were fed with a high-fat diet (HFD; $45 \% \mathrm{kcal}$ fat, D12451, Research Diet, New Brunswick, NJ, USA) for eight weeks to induce obesity. Eight mice were used in each group for each of the animal tests (described below, except for experiments in Fig. 1 and Fig. 6).

\section{Treadmill training}

For exercise adaptation, all mice were conditioned at 10 metres per minute for five days to become familiar with the treadmill environment (LE8710RTS, Harvard Apparatus, Holliston, Massachusetts, USA). After adaptation, mice randomised to the exercise group were subjected to moderate-intensity continuous training corresponding to $65-70 \%$ of $\mathrm{VO}_{2} \mathrm{max}$, where the average running time was close to two hours per day for four weeks. Gentle tapping on the tail or hind limb was given to encourage running if the animal stopped.

\section{Tail suspension test (TST)}

Mice were suspended individually by their tails from a metal rod using adhesive Scotch tape affixed to their tails. The rod was fixed $50 \mathrm{~cm}$ above the surface of a table covered with a soft cloth in a sound-isolated room. The test lasted for six minutes. The immobility time was measured using a stopwatch by an observer who was blind to the experimental design (30).

\section{Forced swimming test (FST)}

Mice were placed in a Plexiglas cylinder (internal diameter $=10$ $\mathrm{cm}$; height $=50 \mathrm{~cm}$ ) filled with $25-26^{\circ} \mathrm{C}$ water $($ water level $=$ $10 \mathrm{~cm}$ ). The test lasted for six minutes and the behaviour of the mice was evaluated during final five minutes. The immobility time was measured using a stopwatch by an observer who was blind to the experimental design. An animal was considered immobile if it remained floating in water, making only movements necessary to keep its head above water (31).

\section{Sucrose performance test (SPT)}

Individually housed mice were simultaneously supplied with one bottle of tap water and another bottle with $2 \%$ (wt/vol) sucrose solution for 24 hours, with positions of the bottles swapped half way through the test (i.e. at the $12^{\text {th }}$ hour). The consumption of water and sucrose solution was measured by the difference in weight of each of the bottles before and after the test. Preference to sucrose was calculated as the percentage of sucrose consumption over total liquid consumption (32).

\section{Open field test (OFT)}

Mice were placed in the centre of a brightly lit (650-700 lux) or dimly lit (20-30 lux) chamber of an open-field apparatus $($ size $=44 \times 44 \times 30 \mathrm{~cm})$. Movements of the animals were tracked 
by an automatic monitoring system (Panlab, Harvard Apparatus, Holliston, Massachusetts, USA) for 20 minutes. Activity in the central area was calculated by dividing the distance travelled in the central area by the total distance travelled (33).

\section{Social interaction test (SIT)}

Social activity of male mice that were naive to each other was tested in a brightly lit, unfamiliar environment. The animals were placed in the opposite areas of a transparent box (size $=44 \times 44 \times 30$ $\mathrm{cm}$ ) and separated by a removable partition. After five minutes of habituation, the partition was removed and the activity of the animals was recorded on videotapes for 10 minutes. Number of social interactions was registered by an observer who was blind to the experimental design (34).

\section{Tissue preparation}

Mice were deeply anesthetised with a mixture of ketamine and xylazine. Upon collection of trunk blood, they were sequentially perfused with $0.9 \%$ saline for five minutes and $4 \%$ (wt/vol) paraformaldehyde (PFA) in $0.1 \mathrm{M}$ Phosphate buffer (PBS) for 15 minutes. Serum, skeletal muscle and hippocampus were collected and stored at $-80^{\circ} \mathrm{C}$ for further analysis.

The isolated brains were post-fixed in $4 \%$ PFA overnight at $4{ }^{\circ} \mathrm{C}$ and then transferred to $30 \%$ (wt/vol) sucrose solution until they sank. The brain slices (1-in-6 series; $40 \mu \mathrm{m}$ thickness) were cryosectioned using a sliding freezing microtome (Thermofisher). The slices were stored in cryoprotectant at $-20^{\circ} \mathrm{C}$ until analysis.

\section{Immunohistochemistry and immunofluorescent staining}

The sections were retrieved in citrate buffer $\left(\mathrm{pH} \mathrm{6.0)}\right.$ at $95^{\circ} \mathrm{C}$ for 30 minutes, followed by incubation in $2 \mathrm{~N} \mathrm{HCl}$ for 30 minutes at $37^{\circ} \mathrm{C}$ and $0.1 \mathrm{M}$ borate buffer (pH 8.5) for 15 minutes at room temperature. After washing in $0.01 \mathrm{M}$ PBS, the sections were incubated overnight with anti-BrdU antibodies (1:1000, Abcam), followed by incubation with biotinylated goat anti-rat $\operatorname{IgG}(1: 200$; Dako). The BrdU staining was visualised with the peroxidase method (ABC system, vector Laboratories) and diaminobenzidine kits (DAB kits, Sigma-Aldrich). For DCX and Ki67 staining, sections were incubated with rabbit anti-DCX (1:200; Abcam) and rabbit anti-Ki67 (1:1000; Novocastra) antibodies respectively, followed by biotinylated goat anti-rabbit $\operatorname{IgG}$ (1:200; Dako) and visualised with the aforementioned method.

\section{Quantitative real-time PCR analysis}

Total RNA from hippocampus, hypothalamus and skeletal muscle was extracted with TRIzol reagent (Invitrogen) and then subjected to reverse transcription and real-time PCR analysis on an ABI 7000 instrument (Applied Biosystems), as described above. The primers used for real-time PCR analysis are listed in Supplementary Table 1.

\section{Western blot analysis}

Total proteins from hippocampus, hypothalamus and skeletal muscle were extracted by RIPA buffer and then subjected to SDSPAGE gel separation. PVDF membrane was probed with primary antibodies for $\beta$-klotho (Cell Signaling Technology, Danvers, MA, USA), FGFR1 (Cell Signaling Technology, Danvers, MA, USA) and Tubulin (Cell Signaling Technology, Danvers, MA, USA). The proteins were detected with enhanced chemiluminescence reagents (GE healthcare) and quantified using the NIH Image $\mathbf{J}$ software.

\section{Statistical analysis}

Experiments were independently-repeated at least three times and data were presented as means $\pm \mathrm{SEM}$; a $p$ value $<0.05$ was considered significant. Statistical analysis was performed with GraphPad Prism 7 (GraphPad Software, La Jolla, CA, USA). Betweengroups comparisons were performed using ANOVA followed by the Tukey's multiple comparison test or the Student's $t$ test where appropriate.

\section{SUPPLEMENTARY MATERIALS}

Supplementary materials for this article are available at http://www. humanbab.net/qfy-content/uploads/2020/04/2020010102ST1.pdf Supplementary Table 1. List of primer sequences used for RT-PCR analysis in this study.

\section{REFERENCES}

1. GBD 2015 Disease and Injury Incidence and Prevalence Collaborators, Global, regional, and national incidence, prevalence, and years lived with disability for 310 diseases and injuries, 1990-2015: A systematic analysis for the global burden of disease study. Lancet 388, 1545 1602 (2016).

2. S. Y. Yau, A. Li, R. L. C. Hoo, Y. P. Ching, B. R. Christie, T. M. C. Lee, A. Xu, K. F. So, Physical exercise-induced hippocampal neurogenesis and antidepressant effects are mediated by the adipocyte hormone adiponectin. Proc. Natl. Acad. Sci. U.S.A. 111, 15810-15815 (2014)

3. S. Y. Yau, B. W. Lau, J. B. Tong, R. Wong, Y. P. Ching, G. Qiu, S. W. Tang, T. M. Lee, K. F. So, Hippocampal neurogenesis and dendritic plasticity support running-improved spatial learning and depression-like behaviour in stressed rats. PloS One 6, 24263 (2011).

4. I. Delgado, L. Huet, S. Dexpert, C. Beau, D. Forestier, P. Ledaguenel, A. Aubert, J. Sauvant, B. Aouizerate, E. Magne, L. Capuron, Depressive symptoms in obesity: Relative contribution of low-grade inflammation and metabolic health. Psychoneuroendocrinology 91, 55-61 (2018).

5. Y. Milaneschi, W. K. Simmons, E. F. C. van Rossum, B. W. Penninx, Depression and obesity: Evidence of shared biological mechanisms. Mol. Psychiatr. 24, 18-33 (2019).

6. M. S. Faith, M. Butryn, T. A. Wadden, A. Fabricatore, A. M. Nguyen, S. B. Heymsfield, Evidence for prospective associations among depression and obesity in population-based studies. Obes. Rev. 12, e438-e453 (2011).

7. M. Gleeson, N. C. Bishop, D. J. Stensel, M. R. Lindley, S. S. Mastana, M. A. Nimmo, The anti-inflammatory effects of exercise: Mechanisms and implications for the prevention and treatment of disease. Nat. Rev. Immunol. 11, 607-615 (2011).

8. Z. Lin, H. Tian, K. S. L. Lam, S. Lin, R. C. L. Hoo, M. Konishi, N. Itoh, Y. Wang, S. R. Bornstein A. Xu, X. Li, Adiponectin mediates the metabolic effects of FGF21 on glucose homeostasis and insulin sensitivity in mice. Cell Metab. 17, 779-789 (2013).

9. C. Greenhill, Neuroendocrinology: Role for adiponectin in effects of exercise in depression Nat. Rev. Endocrinol. 11, 4 (2015).

10. A. Kharitonenkov, T. L. Shiyanova, A. Koester, A. M. Ford, R. Micanovic, E. J. Galbreath, G. E. Sandusky, L. J. Hammond, J. S. Moyers, R. A. Owens, J. Gromada, J. T. Brozinick, E. D. Hawkins, V. J. Wroblewski, D.-S. Li, F. Mehrbod, S. R. Jaskunas, A. B. Shanafelt, FGF-21 as a novel metabolic regulator. J. Clin. Invest. 115, 1627-1635 (2005).

11. G. Singhal, F. M. Fisher, M. J. Chee, T. G. Tan, A. E. Ouaamari, A. C. Adams, R. Najarian, R. N. Kulkarni, C. Benoist, J. S. Flier, Maratos-Flier E., Fibroblast growth factor 21 (fgf21) protects against high fat diet induced inflammation and islet hyperplasia in pancreas. PloS One 11, 148252 (2016)

12. X. Ding, J. Boney-Montoya, B. M. Owen, A. L. Bookout, K. C. Coate, D. J. Mangelsdorf S. A. Kliewer, Betaklotho is required for fibroblast growth factor 21 effects on growth and metabolism. Cell Metab. 16, 387-393 (2012).

13. Z. Lin, X. Pan, F. Wu, D. Ye, Y. Zhang, $Y$. Wang L, Jin, Q Lian, Y. Huang, H. Ding C. Triggle, K. Wang $X, L i, A, X u$, Fibroblast growth factor 21 prevents atherosclerosis by suppression of hepatic sterol regulatory element-binding protein-2 and induction of adiponectin in mice. Circulation 131, 1861-1871 (2015).

14. Q. Liang, L. Zhong, J. Zhang, Y. Wang, S. R. Bornstein, C. R. Triggle, H. Ding, K. S. L. Lam, A. X U, FGF21 maintains glucose homeostasis by mediating the cross talk between liver and brain during prolonged fasting. Diabetes 63, 4064-4075 (2014).

15. B. K. Tan, M. Hallschmid, R. Adya, W. Kern, H. Lehnert, H. S. Randeva, Fibroblast growth factor 21 (FGF21) in human cerebrospinal fluid. Diabetes 60, 2758-2762 (2011).

16. H. H. Chang, P. S. Chen, Y. W. Cheng, T. Y. Wang, Y. K. Yang, R. B. Lu, Fgf21 is associated with metabolic effects and treatment response in depressed bipolar II disorder patients treated with valproate. Int. J. Neuropsychoph. 21, 319-324 (2018).

17. O. Wang J. Yuan, Z, Yu, L. Lin, Y Jiang, Z Cao, P. Zhuang M. J. Whalen, B. Song, X.J. Wang X. Li, E. H. Lo, Y. Xu, X. Wang, FGF21 attenuates high-fat diet-induced cognitive impairment via metabolic regulation and anti-inflammation of obese mice. Mol. Neurobiol. 55, 4702-4717 (2018).

18. A. L. Bookout, M. H. M. de Groot, B. M. Owen, S. Lee, L. Gautron, H. L. Lawrence, X. Ding, J. K. Elmquist, J. S. Takahashi, D. J. Mangelsdorf, S. A. Kliewer, FGF21 regulates metabolism and circadian behavior by acting on the nervous system. Nat. Med. 19, 1147-1152 (2013).

19. H. Hsuchou, W. Pan, A. J. Kastin, The fasting polypeptide FGF21 can enter brain from blood. Peptides 28, 2382-2386 (2007).

20. S. Kvam, C. L. Kleppe, I. H. Nordhus, A. Hovland, Exercise as a treatment for depression: A meta-analysis. J. Affect. Disord. 202, 67-86 (2016).

21. K. H. Kim, S. H. Kim, Y. K. Min, H. M. Yang, J. B. Lee, M. S. Lee, Acute exercise induces fgf21 expression in mice and in healthy humans. Plos One 8, 63517 (2013).

22. Y. Izumiya, H. A. Bina, N. Ouchi, Y. Akasaki, A. Kharitonenkov, K. Walsh, FGF21 is an Akt- 
regulated myokine. FEBS Letters 582, 3805-3810 (2008).

23. Y. Liu, M. Wang, X. Tan, X. Wang, X. Yang, J. Xiao, X. Li, F. Wang, Negative correlation between cerebrospinal fluid fgf21 levels and bdi scores in male chinese subjects. Psychiatr. Res. 252 111-113 (2017)

24. T. Jelenik, M. Dille, S. Müller-Lühlhoff, D. G. Kabra, Z. Zhou, C. Binsch, S. Hartwig, S. Lehr, A. Chadt, E. M. Peters, J. Kruse, M. Roden, H. Al-Hasani, T. R. Castañeda, FGF21 regulates insulin sensitivity following long-term chronic stress. Mol. Metab. 16, 126-138 (2018).

25. C. Vivar, Adult hippocampal neurogenesis, aging and neurodegenerative diseases: Possible strategies to prevent cognitive impairment. Curr. Top. Med. Chem. 15, 2175-2192 (2015).

26. W. L. Holland, A. C. Adams, J. T. Brozinick, H. H. Bui, Y. Miyauchi, C. M. Kusminski, S. M. Bauer, M. Wade, E. Singhal, C. C. Cheng, K. Volk, M.-S. Kuo, R. Gordillo, A. Kharitonenkov, P. E. Scherer, An FGF21-adiponectin-ceramide axis controls energy expenditure and insulin action in mice. Cell Metab. 17, 790-797 (2013).

27. A. D. Kriketos, S. K. Gan, A. M. Poynten, S. M. Furler, D. J. Chisholm, L. V. Campbell, Exercise increases adiponectin levels and insulin sensitivity in humans. Diabetes Care 27, 629-630 (2004).

28. K. A. Simpson, M. A. F. Singh, Effects of exercise on adiponectin: A systematic review. Obesity 16, 241-256 (2008)

29. M. Suman, D. Neha, G. Arun, P. Anand, Estimation of lung cancer burden in Australia, the Philippines, and Singapore: An evaluation of disability adjusted life years. Cancer Biol. Med. 14, 74-82 (2017).

30. L. Steru, R. Chermat, B. Thierry, P. Simon, The tail suspension test: A new method for screening antidepressants in mice. Psychopharmacology 85, 367-370 (1985).
31. R. D. Porsolt, A. Bertin, M. Jalfre, Behavioural despair in mice: A primary screening test for antidepressants. Arch. Int. Pharmacodyn. Ther. 229, 327-336 (1977).

32. L. Z. Agudelo, T. Femenía, F. Orhan, M. Porsmyr-Palmertz, M. Goiny, V. Martinez-Redondo, J. C. Correia, M. Izadi, M. Bhat, I. Schuppe-Koistinen, A. T. Pettersson, D. M. S. Ferreira, A. Krook R. Barres, J. R. Zierath, S. Erhardt, M. Lindskog, J. L. Ruas, Skeletal muscle PGC-1 $\alpha 1$ modulates kynurenine metabolism and mediates resilience to stress-induced depression. Cell 159, 33-45 (2014)

33. V. Carola, F. D'Olimpio, E. Brunamonti, F. Mangia, P. Renzi, Evaluation of the elevated plusmaze and open-field tests for the assessment of anxiety-related behaviour in inbred mice. Behav. Brain Res. 134, 49-57 (2002).

34. K. Tanda, A. Nishi, N. Matsuo, K. Nakanishi, N. Yamasaki, T. Sugimoto, K. Toyama, K. Takao, T. Miyakawa, Abnormal social behavior, hyperactivity, impaired remote spatial memory, and increased D1-mediated dopaminergic signaling in neuronal nitric oxide synthase knockout mice. Mol. Brain 2, 19 (2009).

Acknowledgements: Funding: This study was supported by Hong Kong Health and Medical Research Fund (03144516), National Natural Science Foundation of China (81600660) and General Research Fund of Hong Kong Research Grants Council (17127518). Author contributions: Y.L, S.Y, Q.L and Y.W carried out the experiments. Y.L analysed and interpreted the data and wrote the manuscript. A.X conceived and supervised the study and edited the manuscript. Competing interests: The authors declare that they have no competing interests. Data and materials availability: All data and materials are available upon request to the corresponding author. 